\title{
Modeling technology of radar scattering of the fourth generation EF-2000 Typhoon multipurpose aircraft model
}

\author{
Sergey Herasimov ${ }^{1}$, Yaroslav Belevshchuk ${ }^{2}$, Ivan Ryapolov ${ }^{3}$, Andriy Volkov ${ }^{4}$, \\ Maksym Borysenko ${ }^{5}$, Oleksandr Tokar ${ }^{6}$ \\ ${ }^{1}$ Ivan KozhedubKharkiv National Air Force University, Kharkiv, Ukraine, gsvnr@ukr.net \\ ${ }^{2}$ Ivan KozhedubKharkiv National Air Force University, Kharkiv, Ukraine, i.ryapolov.79@ukr.net \\ ${ }^{3}$ Ivan KozhedubKharkiv National Air Force University, Kharkiv, Ukraine, i.ryapolov.79@ukr.net \\ ${ }^{4}$ Ivan KozhedubKharkiv National Air Force University, Kharkiv, Ukraine, vaf75takt@ gmail.com \\ ${ }^{5}$ Ivan KozhedubKharkiv National Air Force University, Kharkiv, Ukraine, bmaxxss1976@gmail.com \\ ${ }^{6}$ Ivan KozhedubKharkiv National Air Force University, Kharkiv, Ukraine, sash.2015@ukr.net
}

\begin{abstract}
The paper presents a technologyfor calculating the characteristics of an aircraft secondary radiation for the analysis of radar scattering of the fourth generation EF-2000 Typhoon multipurpose fighter. The main characteristics of the aircraft radar profiles are substantiated - the effective scattering surface, its mean and median values, the distribution laws of the amplitude coefficient of the reflected signal. The results of calculating such scattering characteristics of the EF-2000 Typhoon aircraft for the main frequencies of the sounding signal used in the radar are presented. The results obtained are proposed to be used in the modernization and design of advanced radar and aviation facilities. The application of the results obtained will allow to optimize the design of the modernized perspective aircraft in order to reduce the radar signature. The presented results are necessary to assess the capabilities of various design options for radar systems for identification and recognition of aircraft of this type. The results obtained are useful for solving applied problems of aircraft construction and radar.
\end{abstract}

Key words :aerodynamic model of an aircraft, radar scattering, radiation.

\section{INTRODUCTION}

Of the main components in the design of an aircraft is the development of indicators for assessing layout and design solutions. Such indicators should reflect the main purpose of creating the aircraft. The purpose is determined by the range of tasks that the created aircraft solves. The solution of such problems is achieved by finding the optimal combination of flight, unmasking, operational and other characteristics. The combination of such characteristics on the basis of an integrated approach makes it possible to formulate requirements for the appearance of the aircraft [1]. When creating military aircraft, the goal is to ensure a low level of visibility when irradiated by a sounding radio signal from ground and airborne radar stations. To use the indicator of the radar signature of the aircraft, which is characterized by the level of scattering (secondary radiation). This indicator makes it possible to assess the impact of design and layout solutions on the flight performance of the aircraft being created [2].

The parameters data of the aircraft secondary radiation makes it possible to solve a number of practical problems of radar. Firstly, it is possible to carry out a set of measures to form a positional area of subunits to provide reconnaissance and combat information to subunits and units of anti-aircraft missile forces. Secondly, information can be obtained to detect areas on the surface of the aircraft that dominate the backscatter of the aircraft as a whole. This will make it possible to assess the effectiveness of predicted measures to optimize the radar characteristics of both the entire aircraft and its individual sections.

Thus, an urgent scientific task is to calculate the parameters of the secondary radiation of modern aircraft, especially for military purposes.

\section{LITERATURE REVIEW}

The radar profile of an aircraft is understood as a set of radar parameters depending on the sounding signal. The study of radar profiles of aircraft is carried out to improve the indicators of their detection and recognition, when simulating an aircraft of the required type for solving specific problems [1]. The work [2] presents the results of numerical modeling of radar profiles of cruise missiles in the meter, centimeter and decimeter wavelengths. When simulating by numerical methods, the parameters of the secondary radiation of air objects of complex shapes and various electrical sizes were calculated. However, the article focuses on the analysis of long-range radar profiles of cruise missiles at different angles, polarization and spectrum width of the sounding signal; the possibility of using missiles as decoys is being considered. In works [3-5] the disadvantages and advantages of using integral equations for calculating the parameters of secondary radiation of aircraft are substantiated. The presented results can be used only for volumetric objects with small curvature. For example, for cruise missiles or unmanned aerial vehicles $[2,4,6,7]$. 
A method for calculating the effective scattering surface (ESS) of a triangular corner reflector of arbitrary shape and irradiation conditions is presented in [7]. From a practical point of view, the method can lead to difficulties associated with determining the optimal geometric shape of the reflectors and adding active systems for emitting or re-reflecting radar signals.

The need for the study, the results of which are presented in this work, is confirmed by the authors of the articles [8-20]. The solution of a set of tasks associated with an onboard aircraft is possible due to a rational combination of flight technical, unmasking, operational and other characteristics in their totality based on an integrated approach to the formation of its appearance, including when irradiated with various sounding radio technical signals [10].

A feature of this work, in comparison with [11], is the calculation of the radar scattering of aircraft models made using the ESS reduction technology.

An analysis of the literature in the field of researching the radar profile of an aircraft allows us to assert that it is relevant to assess the scattering characteristics of vehicles built using the technology for reducing ESS based on previously created high-frequency calculation methods[12-17].

The aim of the study is to determine the radar characteristics of the EF-2000 Typhoon multipurpose fighter, which is built using ESS reduction technology, at various radiation frequencies. The results of the study will make it possible to optimize the design of modernized and perspective aircraft in order to reduce their radar signature and give recommendations on the use of the necessary radar equipment for the timely detection of such aircraft, including those built using Stealth technology[19].

To achieve the goal of the study, the following tasks were set: to develop a surface model for the EF-2000 Typhoon multipurpose fighter;

to substantiate the features of radio-technical impact (centimeter, decimeter, meter wavelength ranges) on the parameters of the radar characteristics (radar profile) of the EF-2000 Typhoon multipurpose fighter;

to substantiate recommendations on the use of the required radar stations for the timely detection of aircraft designed to reduce the effective scattering surface.

\section{DEVELOPMENT OF TECHNOLOGY FOR MODELING OF RADAR SCATTERING CHARACTERISTICS OF THE FOURTH GENERATION EF-2000 TYPHOON MULTIPURPOSE FIGHTER}

EF-2000 Typhoon Multipurpose Fighter (Figure 1), enlisted in NATO armies. Although this fighter does not belong to the category of stealth aircraft, a number of structural measures have been carried out in its design aimed at reducing the ESS. Even at the beginning of the design, the developers of the aircraft were tasked with reducing the front ESS by four times compared to the PanaviaTyphoon fighter-bomber $[12,13]$. To reduce the reflection of rays from the engine input stages, the air intake inlets are covered with radio absorbent materials. Although the EF-2000 Typhoon does not have internal arms compartments, the external suspensions are semi-submerged, which allows them to be partially shielded from incident electromagnetic radiation. The elements of the aircraft with the highest reflectivity (bearing planes, elements of horizontal plumage) are covered by radio-absorbing materials.

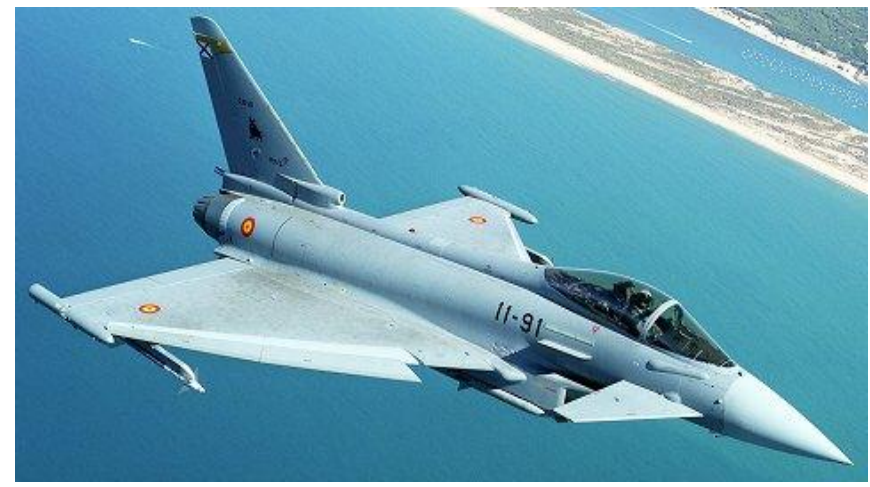

Figure 1: EF-2000 Typhoon multipurpose fighter

The EF-2000 Typhoon ESS is about a quarter of the corresponding PanaviaTyphoon fighter-bomber value. The exact value of the EF-2000 Typhoon ESS is not officially reported, but it is estimated by experts to be less than 1 square meter without external suspensions $[19,20]$.

The main technical characteristics of the EF-2000 Typhoon are as follows $[19,20]$ : length $-15,96 \mathrm{~m}$, wingspan $-10,95 \mathrm{~m}$, height $-5,28 \mathrm{~m}$, weight $-(11000 \ldots 23500) \mathrm{kg}$, engine type 2 turbojets two-circuit engines with afterburner Eurojet EJ 200, maximum speed $-2120 \mathrm{~km} / \mathrm{h}$, flight ceiling $-19812 \mathrm{~m}$, combat radius $-(601 \ldots 1390) \mathrm{km}$.

To calculate the radar parameters of the secondary radiation of the EF-2000 Typhoon (in particular, the ESS), a model of its surface was built (Figure 2).

The modeling of the surface of this aircraft was carried out according to the technique described in $[11,13,18]$. The modeling is based on the results of the study $[6,10]$, the purpose of which is to substantiate laser multifrequency signals for the detection and tracking of aircraft.

In the simulation, the smooth part of the flat surface of the aircraft was approximated by sections of 104 triaxial ellipsoids. Fractures of the aircraft surface were modeled using 10 straight edge scatter plots.

The main characteristic that determines the properties of an aircraft reflecting electromagnetic waves is $\operatorname{ESS}[7,8,11]$.

The main correlations of the method of obtaining radar characteristics of an aircraft are considered in publications [7, $8,11]$.Aircraft sounding is carried out in a flat monochromatic Electromagnetic wave (EMW) with a single amplitude with polarization and direction of propagation (Figure 2).

The secondary scattering parameters for Typhoon EF-2000 were estimated using the following values:

sounding elevation angle -3 degrees relative to the horizon plane (sounding from the lower hemisphere);

the step of changing the azimuth of sounding 0,02 degrees; azimuth is measured in degrees from the nose angle of the aircraft ( 0 degrees - nose sounding, 180 degrees - tail sounding);

polarization horizontal.

When simulating, the following results were obtained: 
effective scattering surface pie charts and "incoherent" effective scattering surface;

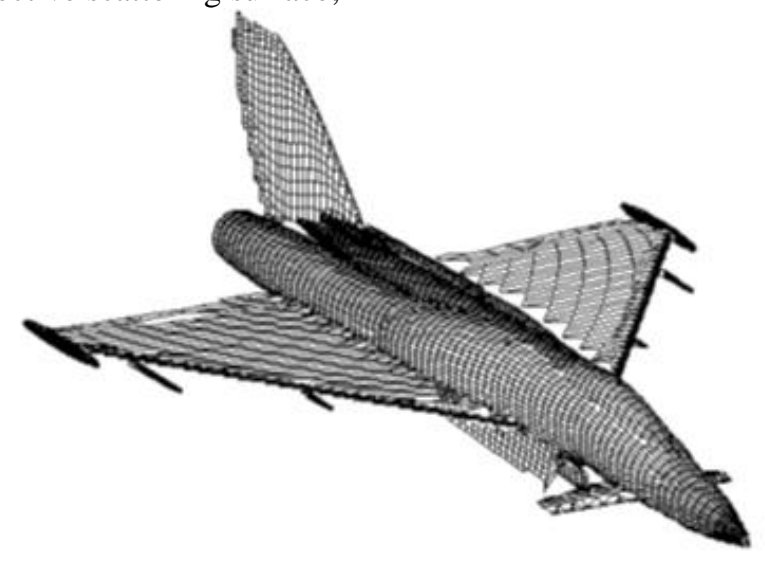

Figure 2:A model of the EF-2000 Typhoon multipurpose fighter

When simulating, the following results were obtained: effective scattering surface pie charts and "incoherent" effective scattering surface;

average and median estimates of the effective scattering surface for the main ranges of azimuths of irradiation with a radio technical signal: nose part $(0 . . .45$ degrees $)$; side part (45...135 degrees); tail section (135...180 degrees); mean and median effective scattering surface estimates for 20-degree irradiation azimuth ranges.

The median value for evaluating the effective scattering surface in a certain sector of sounding angles is understood as some non-random value, the probability of exceeding or not exceeding which in a given sector of angles is 0,5.

"Incoherent" effective scattering surface (IESS) refers to the sum of the ESS of individual surface regions, which does not take into account mutual phase shifts.

The results are given for different frequencies of radiation of a sounding probing radio-technical signal.

\subsection{Radar characteristics of the EF-2000 Typhoon at $10 \mathrm{GHz}(3 \mathrm{~cm}$ wavelength)}

Figure 3 shows a pie chart of ESS for theEF-2000 Typhoon model.

Figure 4 shows a pie chart of IESS for the EF-2000 Typhoon model. The IESS value is a good and sufficiently robust estimate of the ESS over a certain range of probing frequencies and target angles. ESS values are given in $\mathrm{m}^{2}$. An average ESS for the EF-2000 Typhoon model is $18,29 \mathrm{~m}^{2}$. A circular median ESS (a value for ESS, which is used when calculating the detection range of an aircraft with a probability of 0,5 ) is $2 \mathrm{~m}^{2}$.

Figures 5, 6 shows the average and median values of ESS for the basic ranges of irradiation azimuths (nose, side, tail) and for ranges of 20 degrees. ESS values are given in $\mathrm{m}^{2}$. Hence, the radiolocation characteristics of the EF-2000 Typhoon model at irradiation frequency of $10 \mathrm{GHz}$ (wavelength is $3 \mathrm{~cm}$ ) are shown in Figures 3-6. The patterns derived make up a radiolocation profile of the EF-2000
Typhoon at a frequency of $10 \mathrm{GHz}$.

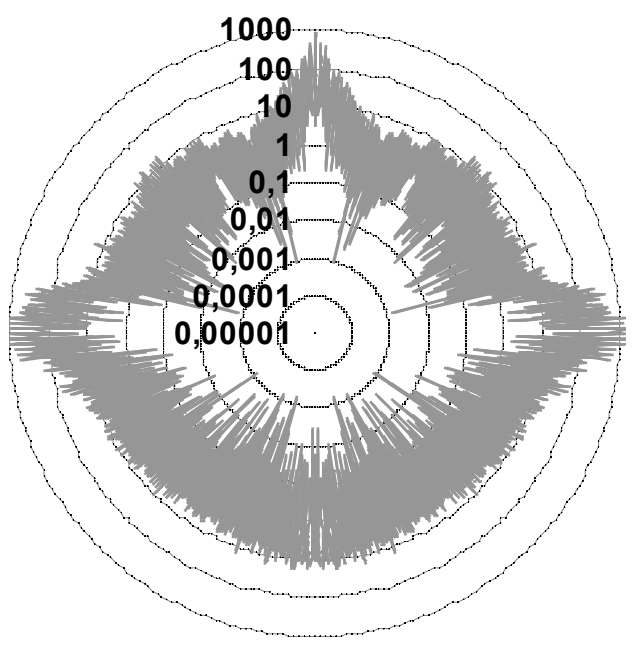

Figure 3:Pie chart of effective scattering surface for theEF-2000 Typhoon multipurpose fighter model (3 cm wavelength)

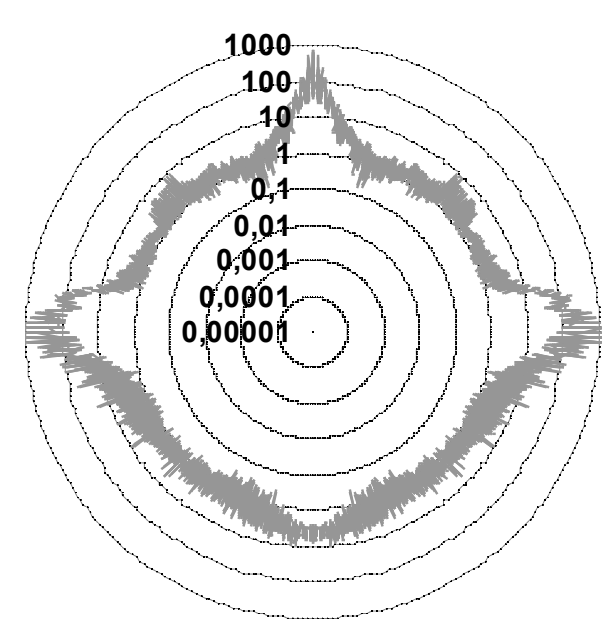

Figure 4: Pie chart of the "non-coherent" effective scattering surface for the EF-2000 Typhoon multipurpose fighter model( $3 \mathrm{~cm}$ wavelength)

\subsection{Radar characteristics of the EF-2000 Typhoon at $1 \mathrm{GHz}(30 \mathrm{~cm}$ wavelength)}

Figure7 shows a pie chart of ESS for the EF-2000 Typhoon model. Figure8 shows a pie chart of IESS for the EF-2000 Typhoon model.

An average ESS for the EF-2000 Typhoon model is 12,95 m² a circular median ESS is $1,45 \mathrm{~m}^{2}$.

Figures 9, 10 shows the average and median values of ESS for the basic ranges of irradiation azimuths (nose, side, tail) and for ranges of 20 degrees. 


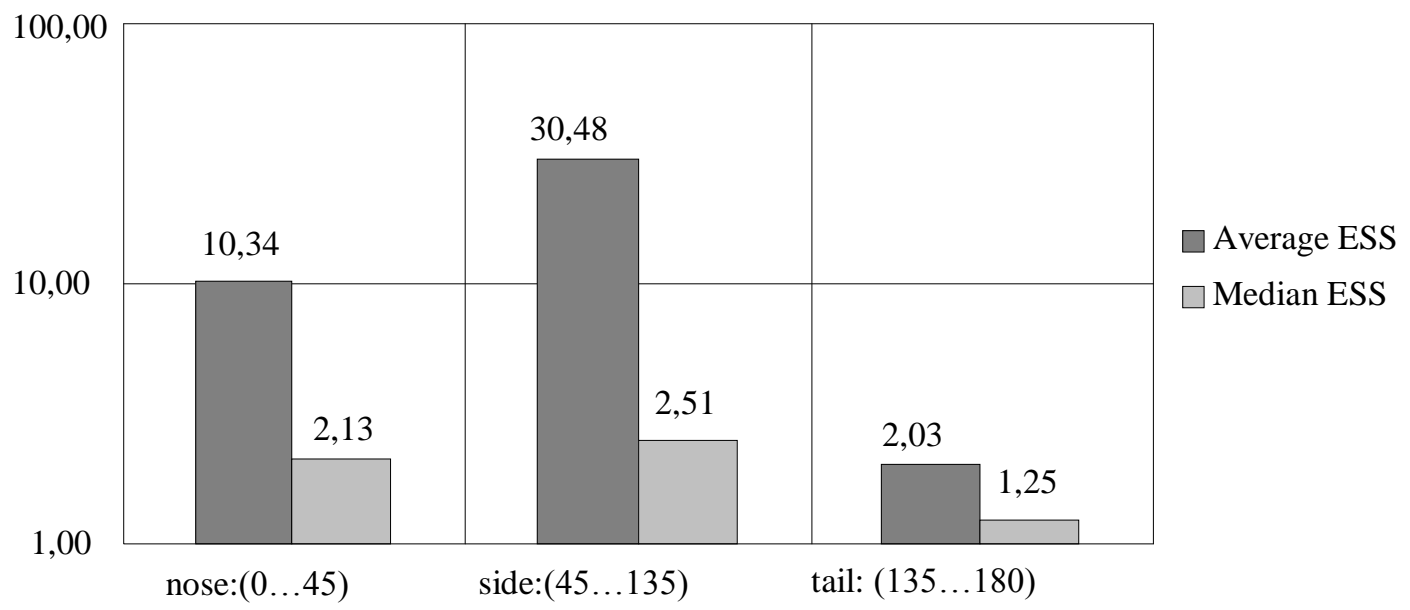

Degrees

Figure 5: Average and median values of effective scattering surface for the basic ranges of irradiation azimuths (nose, side, tail) (3 cm wavelength)

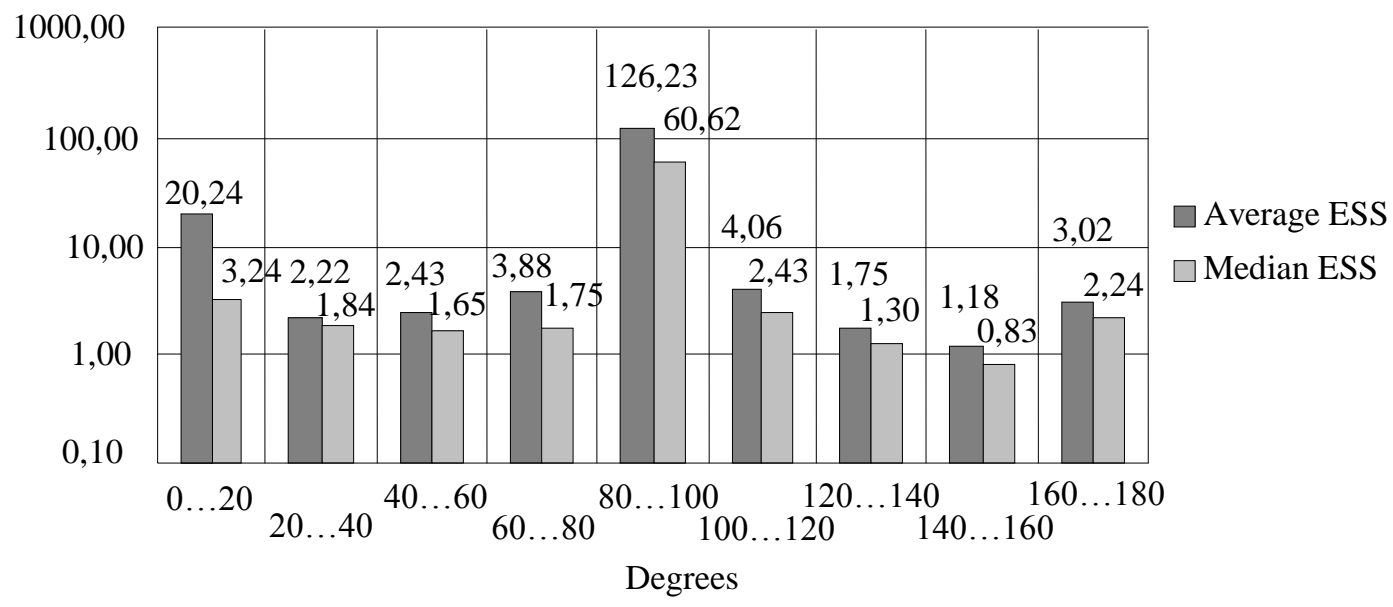

Figure 6: Average and median values of effective scattering surface for the twenty-degree ranges of irradiation azimuths (3 cm wavelength)

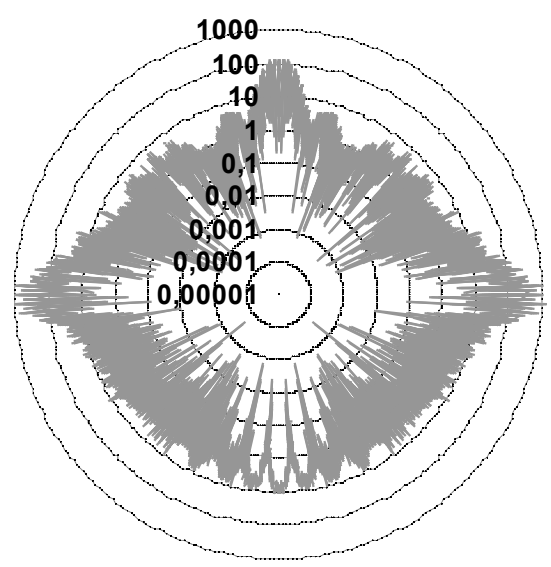

Figure 7:Pie chart of effective scattering surface for the EF-2000 Typhoon multipurpose fighter model (30 cm wavelength)

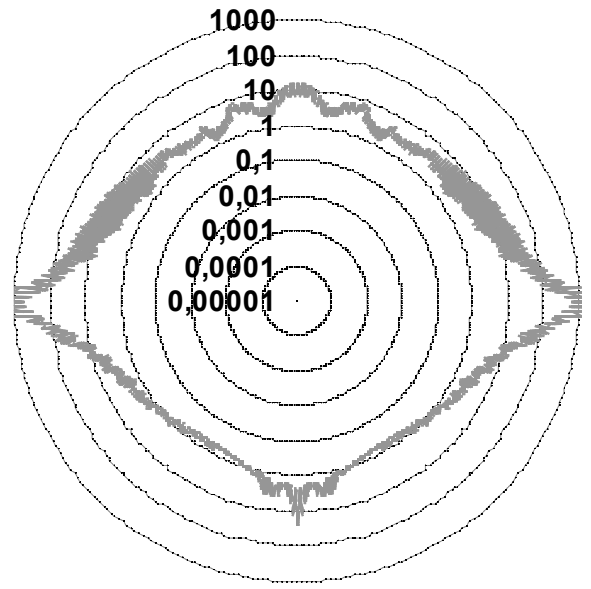

Figure 8: Pie chart of the "non-coherent" effective scattering surface for the EF-2000 Typhoon multipurpose fighter model (30 cm wavelength) 


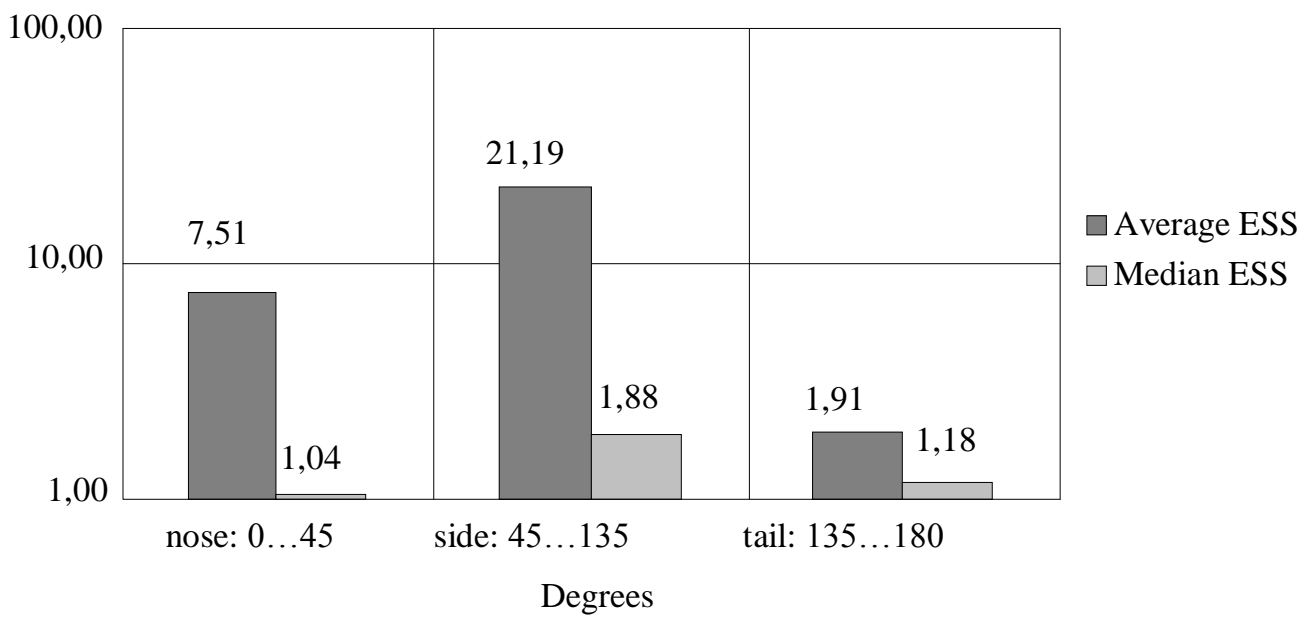

Figure9:Average and median values for the "non-coherent" effective scattering surface for the basic ranges of irradiation azimuths (nose, side, tail)(30 cm wavelength)

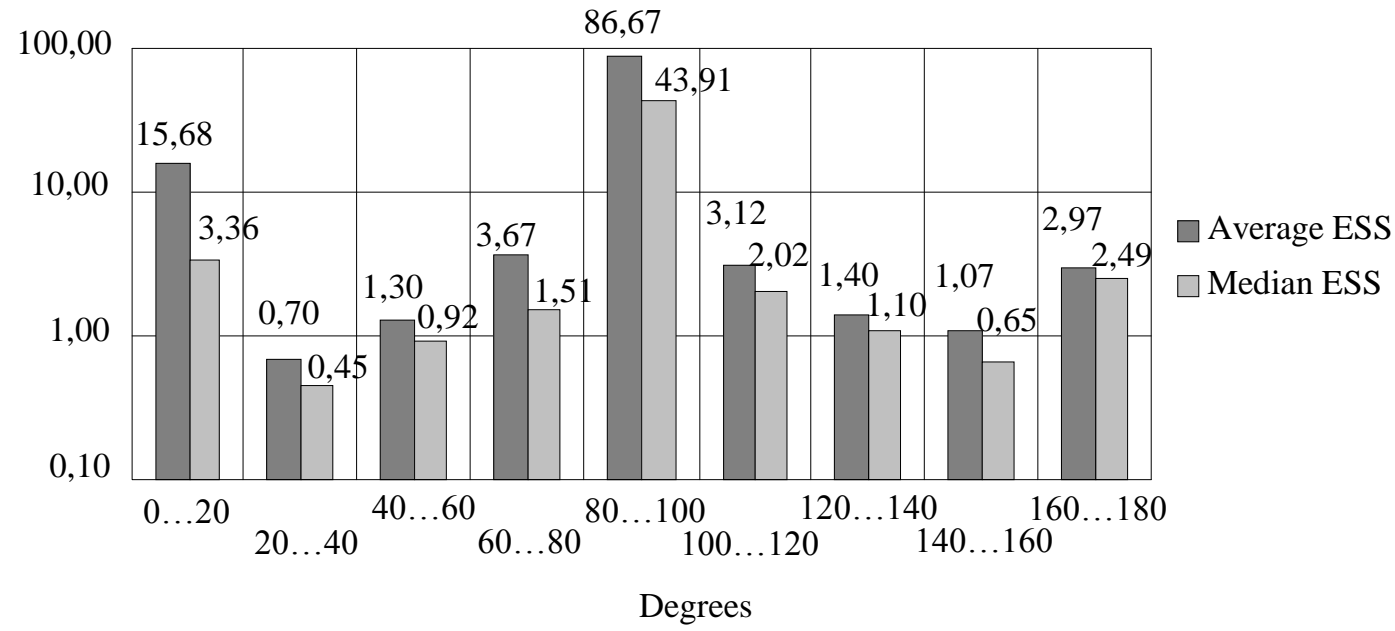

Figure 10:Average and median value of effective scattering surface for the twenty-degree ranges of irradiation azimuths (30 cm wavelength)

Thus, the radiolocation characteristics of the EF-2000 Typhoon model at irradiation frequency irradiation of $1 \mathrm{GHz}$ (wavelength is $30 \mathrm{~cm}$ ) are shown in Fig. 7-10. The patterns derived make up a radiolocation profile for the EF-2000 Typhoon aircraft at a frequency of $1 \mathrm{GHz}$.

\subsection{Radar characteristics of the EF-2000 Typhoon model at a radiation frequency of $166 \mathrm{MHz}(1,8 \mathrm{~m}$ wavelength)} Figure 11 shows a pie chart of ESS for the EF-2000 Typhoon model. Figure 12 shows a pie chart of NESS for the EF-2000 Typhoon model.

An average ESS of the EF-2000 Typhoon model is $13,45 \mathrm{~m}^{2}$; a circular median ESS is $0,80 \mathrm{~m}^{2}$.

Figures 13, 14 shows the average and median values of ESS for the basic ranges of irradiation azimuths (nose, side, tail) and for ranges of 20 degrees.

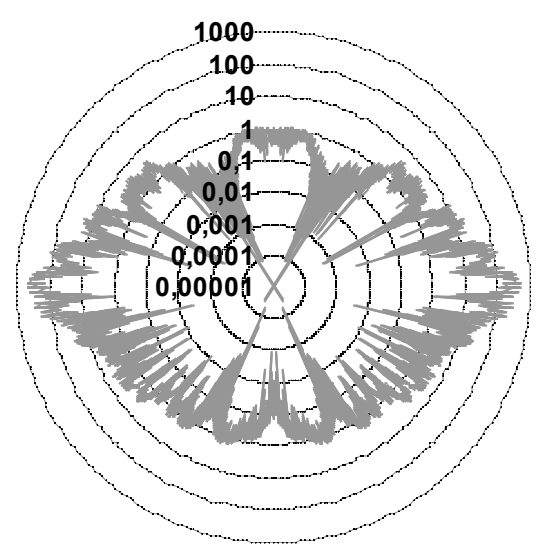

Figure 11:Pie chart of effective scattering surface for the EF-2000 Typhoon multipurpose fighter model (1,8 m wavelength) 


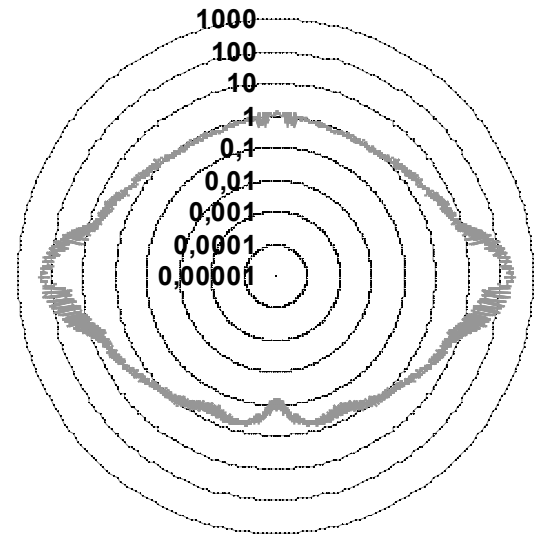

Figure 12:Pie chart of the "non-coherent" effective scattering surface for the EF-2000 Typhoon multipurpose fighter model (1,8 m wavelength)
Hence, the radiolocation characteristics of the EF-2000 Typhoon model at irradiation frequency of $166 \mathrm{MHz}$ (wavelength is $1,8 \mathrm{~m}$ ) are shown in Figures 11-14. The patterns derived make up a radiolocation profile for the EF-2000 Typhoon at a frequency of $166 \mathrm{MHz}$.

\section{RESULTS AND DISCUSSIONS}

A model has been developed for evaluating the parameters of the secondary radiation of an EF-2000 Typhoon aircraft irradiated with radio technical signals of different frequencies. The characteristics scattering assessment of electromagnetic waves on complex bodies, partially covered with radio-absorbing materials, implies taking into account the geometric dimensions.

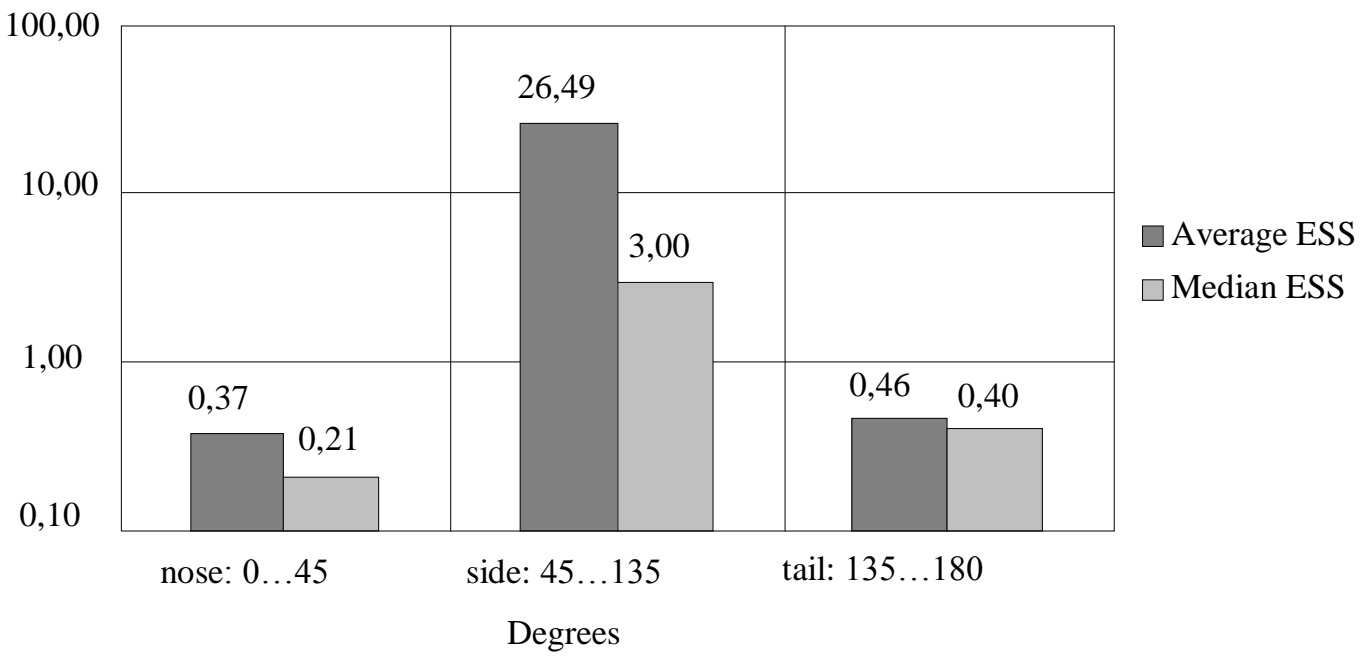

Figure 13:Average and median values of effective scattering surface for the basic ranges of irradiation azimuths (nose, side, tail)(1,8 m wavelength)

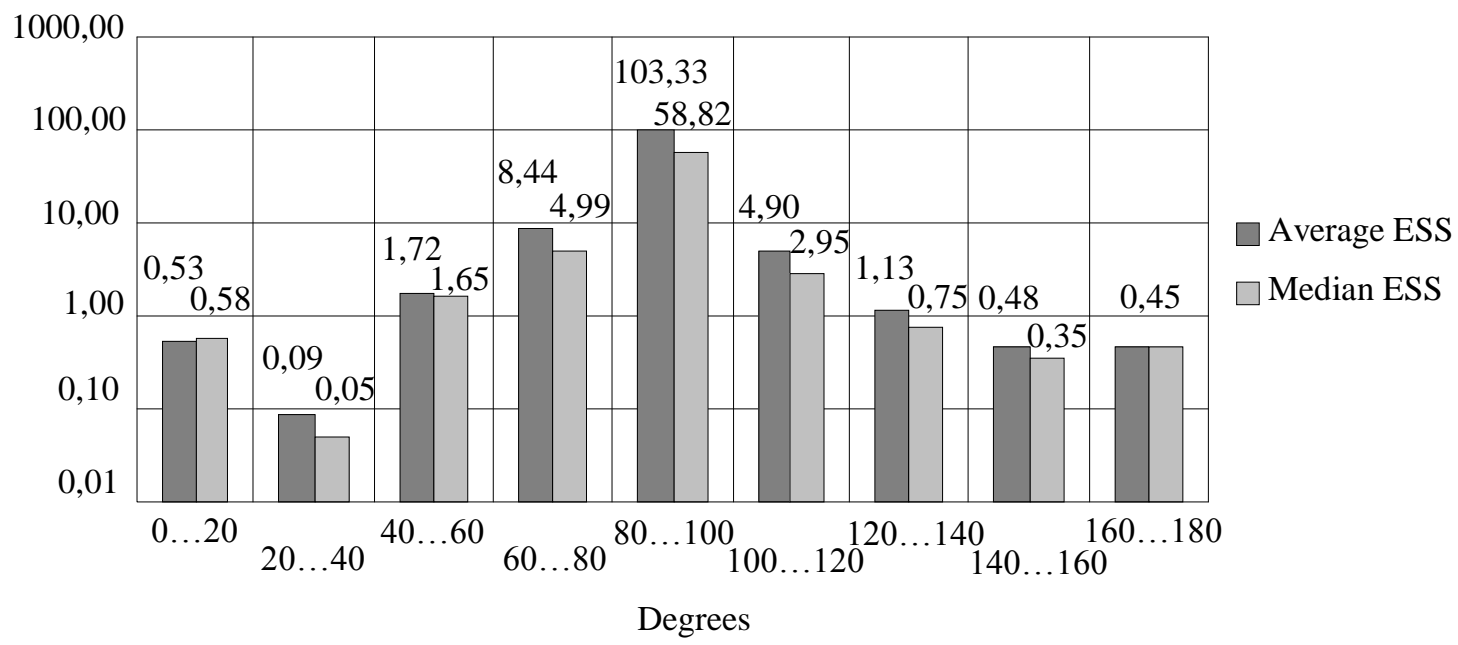

Figure 14:Average and median values of effective scattering surface for the twenty-degree ranges of irradiation azimuths (1,8 m wavelength) 
It is shown that the radar characteristics of such objects differ significantly from analogs of the corresponding geometric dimensions. However, the issues related to the construction of radar profiles of aircraft manufactured using the ESS reduction technology have not yet been resolved. The reason for this may be objective difficulties associated with the lack of an exhaustive consideration of the entire set of prevailing factors. A variant of overcoming the corresponding difficulties could be the construction of mathematical models of the electromagnetic waves scattering on complex bodies using elementary surfaces. This is the approach used in this work.

The advantage of this study is to obtain scatter characteristics for the EF-2000 Typhoon aircraft based on previously developed high-frequency calculation methods that comprehensively take into account the entire set of prevailing factors.

During the study, a model of the surface of the EF-2000 Typhoon aircraft was built, taking into account the geometric dimensions and the presence of many internal surfaces, which is typical for devices manufactured using the ESS reduction technology. The model consists of smooth surfaces and local edge areas, and also includes internal surfaces for the installation of semi-submersible aircraft weapons. However, the simulation does not take into account the internal (electromagnetic field of the aircraft during flight due to aircraft radiation) and external (electromagnetic fields caused by interaction with the environment during flight) effects. These effects can be taken into account only when carrying out a full-scale experiment. However, it should be noted that, as a rule, the ESS value for combat aircraft is given without taking into account the attached aircraft weapons. Comparison of simulation and experimental results will make corresponding changes to the proposed model.

Further development of the study is proposed to make the necessary changes to the proposed model and modeling principles, taking into account (theoretical justification) of internal and external influences during flight on the aircraft radar and the presence of radio-absorbing materials, especially for stealth.

All this gives grounds to claim to improve the efficiency of aircraft detection and recognition.

Research on this topic is useful for radar portraits of military aviation, including drones.

\section{CONCLUSIONS}

1. The result of the research is the constructed model of the surface of the EF-2000 Typhoon aircraft. It is shown that it is necessary to divide the surface of the plane into two groups: the smooth part of the surface and the edge regions of local scattering. In the simulation, the smooth part of the aircraft surface was approximated using sections of 104 triaxial ellipsoids. Surface breaks were modeled using 10 local forward edge scattering regions.

2. The features of the influence of radiation (centimeter, decimeter, meter wavelength ranges) on the analysis of the radar characteristics (radar profile) of the EF-2000 Typhoon aircraft have been established. Thus, for the centimeter wavelength range, the average effective scattering surface of the EF-2000 Typhoon aircraft model is $18,29 \mathrm{~m}^{2}$, and the median circular ESS is $2,00 \mathrm{~m}^{2}$. For the decimeter wavelength range of irradiation, the average ESS for the EF-2000 Typhoon aircraft model is $12,95 \mathrm{~m}^{2}$, the circular median ESS is $1,45 \mathrm{~m}^{2}$. When irradiated in the meter wavelength range, the average ESP for the model of the EF-2000 Typhoon aircraft is $13,45 \mathrm{~m}^{2}$, the circular median ESS is $0,80 \mathrm{~m}^{2}$.

3. In radar, the mean ESS is used to calculate the aircraft detection range with a probability of 0,5 . Thus, the results of our study show that the EF-2000 Typhoon is most noticeable when irradiated with a centimeter-range radar signal. At the same time, in comparison with previous calculations for an aircraft without the use of ESS reduction technology (Su-25T aircraft [11]), the radar signature has decreased by at least 2 times. However, it should be noted that the EF-2000 Typhoon ESS experts stated that an area less than $1 \mathrm{~m}^{2}$ is only intended for irradiation with a wavelength meter. Therefore, for the timely detection of aircraft, it is proposed to use centimeter radar equipment, made according to the technology of reducing the effective scattering surface. This demonstrates the possibility of applying the results of our research.

\section{ACKNOWLEDGEMENT}

This work was supported by the Ivan KozhedubKharkiv National Air Force University (Kharkiv, Ukraine).

\section{REFERENCES}

1. O.I. Sukharevsky, V.A. Vasilets, and G.S. Zalevsky, Electromagnetic wave scattering by aerial and ground radar objects,2015IEEE Radar Conference, 2015, https://doi.org/10.1109/radar.2015.7130989.

2. G.S.Zalevskiy, V.A.Vasilets, and O.I.Sukharevskiy, Radar range profiles of cruise missiles in different wave bands,Applied Radio Electronics, № 13 (1), 2014, p.p. 20-28.

3. S.Yan, J.-M.Jin, and Z. Nie, Improving the Accuracy of the Second-Kind Fredholm Integral Equations by Using the Buffa-Christiansen Functions, 2011IEEE Transactions on Antennas and Propagation, № 59 (4), 2011,

p.p. 1299-1310,https://doi.org/10.1109/tap.2011.210936 4.

4. W.C.Chen, and N.V.Z.Shuley, Robust Target Identification Using a Modified Generalized Likelihood Ratio Test, 2014IEEE Transactions on Antennas and Propagation, № 62 (1), 2014, p.p. 264-273,https://doi.org/10.1109/tap.2013.2287019.

5. J.M. Munoz-Ferreras, and F.Perez-Martinez, On the Doppler Spreading Effect for the Range-Instantaneous-Doppler Technique in Inverse Synthetic Aperture Radar Imagery,2010IEEE 
Geoscience and Remote Sensing Letters, № 7 (1), 2010, p.p. 180-184, https://doi.org/10.1109/lgrs.2009.2030372.

6. S. Herasimov, O. Tymochko, O. Kolomiitsev, G. Aloshin, O. Kriukov, O. Morozov, and V. Aleksiyev, Formation Analysis Of Multi-Frequency Signals Of Laser Information Measuring System, EUREKA: Physics and Engineering, vol. 5, 2019, p.p. 19-28, https://doi.org/10.21303/2461-4262.2019.00984.

7. O.I.Sukharevsky, V.A.Vasilets, and S.V. Nechitaylo,Scattering characteristics computation method for corner reflectors in arbitrary illumination conditions,2015 International Conference on Antenna Theory and Techniques (ICATT), 2015,https://doi.org/10.1109/icatt.2015.7136836.

8. P.V.Nikitin, S.A.Borisov, andS.V.Dobrovol'skiy, Yu.I. Glukhovskaya, Mathematical model of supersonic heterogeneous in-flow process on a flat obstacle,Surface. X-ray, synchrotron and neutron studies, $\quad$ № $\quad 10, \quad 2016, p . p .50-55$, https://doi.org/10.7868/s0207352816100164.

9. A.V.Gomzin, S.A.Mikhailov, and D.S.Gushchina, Evalution of the State and Development of Aerial Targets Contemporary and Promising Arms Systems, Russian Aeronautics. Aircraft Equipment, № 4, 2008, p.p. 3-6.

10. Y. Kozhushko, D. Karlov, O. Klimishen, M. Bortsova, S. Herasimov, O. Hrichanuk, and V. Bykov, Comparison of the Efficiency of Some Images Superposition Algorithms Used in Aircraft Map-Matching Navigation Systems, 2018 IEEE International Conference on Mathematical Methods in Electromagnetic Theory, 2018, p.p. 282-285.

11. S. Herasimov, Y. Belevshchuk, I. Ryapolov, O. Tymochko, M. Pavlenko, O. Dmitriiev, M. Zhyvytskyi, and N. Goncharenko, Characteristics of radiolocation scattering of the $S U-25 T$ attack aircraft model at different wavelength ranges, Information and controlling systems, Eastern-European Journal of Enterprise Technologies, № 6/9 (96), 2018, p.p. 22-29, https://doi.org/10.15587/1729-4061.2018.152740.

12. Doug Richardson,Stealth Warplanes: Deception, Evasion and Concealment in the Air, London, Salamander,2001, $113 \mathrm{p}$.

13. S. Herasimov, M. Pavlenko, E. Roshchupkin, M. Lytvynenko, O. Pukhovyi, and A. Salii, Aircraft flight route search method with the use of cellular automata, International Journal of Advanced Trends in Computer Science and Engineering, vol. 9, is. 4, 2020, p.p. 5077-5082, https://doi.org/10.30534/ijatcse/2020/129942020.

14. S. Herasimov, E. Roshchupkin, V. Kutsenko, S. Riazantsev, and Yu. Nastishin, Statistical analysis of harmonic signals for testing of Electronic Devices, International Journal of Emerging Trends in Engineering Research, vol.8, is. 7, 2020, p.p. 3791-3798, https://doi.org/10.30534/ijeter/2020/143872020.

15. S. Herasimov, Y. Kozhushko, E. Roshchupkin, V. Dekadin, V. Djus, and Y. Melenti, Evaluation of surface profile of holographic diffraction reflective coatings on scattering chart using in laser alarm systems, International Journal of Emerging Trends in Engineering Research, vol.8, is. 8, 2020, p.p. 4502-4507, https://doi.org/10.30534/ijeter/2020/74882020.

16. A. Alimpiev, P. Berdnik, N. Korolyuk, O. Korshets, and M. Pavlenko, Selecting a model of unmanned aerial vehicle to accept it for military purposes with regard to expert data, Eastern-European Journal of Enterprise Technologies, № 1/9 (85), 2017, p.p. 53-60, https://doi.org/10.15587/1729-4061.2017.93179.

17. S. Herasimov, V. Pavlii, O. Tymoshchuk, M.Yu. Yakovlev, D.Ye. Khaustov, Ye. Ryzhov, L. Sakovych, and Yu.A. Nastishin, Testing Signals for Electronics: Criteria for Synthesis, Journal of Electronic Testing, vol. 35, is. 148, 2019, p.p. 1-9, https://doi.org/10.1007/s10836-019-05798-9.

18. S.V.Herasimov, O.I.Timochko, and S.I.Khmelevskiy, Synthesis method of the optimum structure of the procedure for the control of technical status of complex systems and complexes,Scientific Works of Kharkiv National Air Force University, № 4 (53), 2017, p.p. 148-152.

19. Eurofighter Typhoon - Werkstoffe, Aerodynamik, Flugsteuerung. URL:

http://www.bmlv.gv.at/truppendienst/ausgaben/artikel.p hp?id=752.

20. EF-2000 Typhoon. URL: http://www.airwar.ru/enc/fighter/ef2000. 